\title{
Research on Modeling and Simulation of SI Engine for AFR Control Ap- plication
}

\author{
Meng Lei ${ }^{1}$, Zeng Chunnian ${ }^{2}$, Liang Hong ${ }^{2, *}$, Luo Jie ${ }^{2}$, Luo Wen ${ }^{1}$ and Li Xianghua ${ }^{2}$ \\ ${ }^{1}$ School of Information Engineering, Wuhan University of Technology, Wuhan, Hubei, 430070, China \\ ${ }^{2}$ School of Automation, Wuhan University of Technology, Wuhan, Hubei, 430070, China
}

\begin{abstract}
Accurate AFR control with TWC is a significant method to reduce the exhaust emission of SI engines. To follow the up to date model-based methodology in automotive industries, a virtual engine simulation platform was carried out to simulate a SGMW B15 engine based on enDYNA and improved by adding the AFR path dynamic models. Experiments and simulation results were compared for the model validation both about the engine performance at steady state, and especially the AFR path transient response. Also a soft ECU model was designed for the control algorithm implementation. Simulation results shows that the engine model is appropriate for simulating SI engine operated at steady and transient state, and the close-loop PID controller has better performance for suppressing the overshoot of AFR signal during transient throttle position varying. The simulation model described in this work could support the AFR control algorithm development as a proper virtual engine control objects.
\end{abstract}

Keywords: MVEM, engine modeling, AFR, enDYNA.

\section{INTRODUCTION}

As the sustained growth of automotive production, vehicle tail-pipe exhaust has become one of the major source of the air pollutants. Corresponding emission regulations become increasingly stringent in the countries all around the world for more efficient and lower emission automobiles. The combination of EFI (electronic fuel injection) and TWC (three way catalyst) technologies have been developed during the last two decades to meet the strict emission requirements for conventional SI (spark ignition) engines. Thus, the AFR (air to fuel ratio) needs to be maintained at the stoichiometric value to guarantee the maximum TWC efficiency for simultaneous reduction of $\mathrm{NOx}$ (nitrogen oxides), $\mathrm{CO}$ (carbon monoxide) and $\mathrm{HC}$ (hydrocarbons) to nitrogen, oxygen, carbon dioxide and water [1], meaning that the complete combustion of both oxygen and fuel has occurred in the engine cylinders. However, the accurate AFR control has been extensively investigated over many years [1-3] and is still a challenge because of the nonlinearities, time delays, parameter varying and uncertain characteristics existed in the engine dynamics.

Generally, the AFR control consists of two main parts: the estimate of the air mass; the fuel calculation and injection control. The air mass flow is a passive control parameter which is affected by the gas pedal position following the driver's intension. As a consequence, fuel injection quantity is often controlled by the FPW (fuel injection pulse width) to meet the AFR regulation requirements at different operation point which is determined by the engine load and velocity. AFR control strategies are always based on the analytical engine model which describes the process dynamics. Different from the complex CFD (computational fluid dynamics) models which can provide the information of engine combustion performance, MVEM (mean value engine model) is suitable for real-time simulation and has been widely used in engine control applications $[4,6]$.

The proper analytical MVEM was found by Hendricks first [5], and the essence of MVEM was to describe the physical engine dynamics on the time scale of several engine events without the cycle-to-cycle characteristics. Later investigators expanded the model to the AFR control application such as observer design, control method analysis [7], transient fuel film compensation and also as the control object in simulation [8]. Furthermore, the commercial engine simulation software tools such as dSPACE and DYNAware are also based on the MVEM theory, and widely used by the automotive industries in HIL (hardware-in-the-loop) which is a vital test in the designing process of modern automotive electronic control system [9]. However, the AFR path dynamics and sensor characteristics are omitted in MVEM and HIL simulation. In order to research accurate AFR control algorithms following the modern model-based methodology, there is a need to develop a proper engine dynamic model with the characteristics of AFR path which could be used in the controller design process.

In this work, the MVEM theory and the dynamics of AFR control path are analyzed for the immeasurable parameter observation and controller design. The HIL simulation engine model based on enDYNA is improved by adding the 
transient AFR path dynamics, and the model is matched with the experiment data from the real engine test. Improving the enDYNA model rather than Hendricks's MVEM is a beneficial solution for implementing the model on the real-time simulation platform, because commercial engine model is always comprehensive and superior. Then, a virtual engine test bench is established and the offline or real-time simulation of the SI engine can be used for the engine AFR controller development to optimize fuel economy and reduce emissions.

The paper is organized as follows. The detail modelling of MVEM and AFR path dynamics is discussed in Section 2. Taking the specific B15 engine as example, Section 3 presents a virtual test bench based on the improved enDYNA engine model and the validation by the simulated and measured engine data. The basic AFR control strategy is described in Section 4, and the simulation results is conducted. Finally, conclusions and further recommendations are included in Section 5.

\section{SYSTEM MODEL FOR AFR DYNAMICS}

Since the MVEM describes the mathematical subsystem models for the engine dynamic behaviors which are always used for the control applications, in this section, the AFR dynamics is analyzed based on MVEM. The MVEM consists of three dynamics subsystems: fuel vapor and film, manifold air mass flow, crank shaft and loading [6], and these subsystems can be used in the AFR control for the estimate of the air mass, the fuel injection calculation and the definition of operation points. Furthermore, the transmission and response characteristics of the AFR path are also significant for the controller design and will be discussed in this section.

\subsection{Fuel Vapor and Film Model}

As the wall-wetting phenomenon discussed by Hendricks [2], a part of the fuel out from the injector nozzle would form fuel film on the intake manifold and the other would be as vapor flow. The total fuel together with the air mass into the engine's cylinder is not measurable and unequal to the injected fuel at each engine event, it consists of the fuel vapor flow both from the injector directly and the fuel film evaporation. The model can be expressed in equation form as:

$$
\left\{\begin{array}{l}
\dot{m}_{f v}(t)=(1-X) \dot{m}_{f c m d}(t) \\
\ddot{m_{f f}}(t)=\frac{1}{\tau_{f}}\left(X \cdot \dot{m}_{f c m d}(t)-\dot{m}_{f f}(t)\right) \\
\dot{m_{f c y l}}(t)=\dot{m}_{f v}(t)+\dot{m}_{f f}(t)
\end{array}\right.
$$

Where the fraction of the fuel flow become film is defined as $\mathrm{X}$, and $\tau_{f}$ is the fuel film evaporation time constant. $\dot{m}_{f c y l}$ denotes the fuel mass flow entering the cylinder, $\dot{m}_{f c m d}$ denotes the injected fuel mass flow followed the control command, $\dot{m}_{f v}$ and $\dot{m}_{f f}$ denote the fuel mass flow from the injection directly and the wall film evaporation respectively. All the unit of the fuel mass flow is g per sec. This model is a convenient approximation to the true physical dynamic of the fuel in the engine. Furthermore, the equations can be expressed as a single transfer function:

$$
D(s)=\frac{\dot{M_{f c y l}}(s)}{M_{f c m d}(s)}=\frac{1+(1-X) \tau_{f} S}{1+\tau_{f} s}
$$

Where $\mathrm{s}$ is the Laplace operator.

\subsection{Intake Manifold Air Flow Model}

Familiar with the fuel, the air mass entering the cylinder intake valves $\left(\dot{m}_{a p}\right)$ which will strongly affect the AFR is unmeasurable and unequal to the air mass intake from the throttle $\left(\dot{m}_{a t}\right)$. The difference between $\dot{m}_{a t}$ and $\dot{m}_{a \mathrm{p}}$ is the air mass flow in the manifold $\left(\dot{m_{a}}\right)$ :

$\dot{m}_{a}(t)=\dot{m}_{a t}(t)-\dot{m}_{a p}(t)$

Based on the ideal gas law:

$$
\dot{m}_{a}(t)=\frac{d}{d t}\left(\frac{p_{m} V_{m}}{R T_{m}}\right)
$$

Where $V_{m}$ is the volume of the intake manifold, $R$ is the gas constant of fresh $\operatorname{air}\left(\frac{J}{\mathrm{~kg} \cdot K}\right), T_{m}$ is the manifold air temperature (degrees Kelvin), $p_{m}$ is the manifold air pressure(bar) which can be measured by MAP(manifold absolute pressure).

The air flow across the throttle was physically modelled as two seperated parallel isentropic flows in [10], it can be expressed as:

$$
\left\{\begin{array}{l}
\dot{m}_{a t}(t)=\dot{m}_{a t 1} \frac{p_{a}}{\sqrt{T_{a}}} \beta_{1}(\alpha) \beta_{2}\left(p_{r}\right) \\
\beta_{1}=1-a_{1} \cos (\alpha)+a_{2} \cos ^{2}(\alpha) \\
\beta_{2}=\left\{\begin{array}{c}
\frac{1}{p_{n}}\left(\sqrt{p_{r}^{p_{1}}-p_{r}^{p_{2}}}\right) \quad, \text { if }\left(p_{r} \geq p_{c}\right) \\
1 \quad, \text { if }\left(p_{r}<p_{c}\right) \\
p_{c}=\left(\frac{p_{1}}{p_{2}}\right)^{\left(\frac{1}{p_{2}-p_{1}}\right)} ; p_{n}=\sqrt{p_{c}^{p_{1}}-p_{c}^{p_{2}}}
\end{array}\right.
\end{array}\right.
$$

Where $\dot{m}_{a t 1}$ is a fitting constant, $\alpha$ is the throttle angle (degree), $a_{1}, a_{2}, p_{1}, p_{2}, p_{n}, p_{c}$ are constant parameters which have been found in the paper [10], $p_{r}=p_{\text {man }} / p_{a}$ and $p_{a}$ 


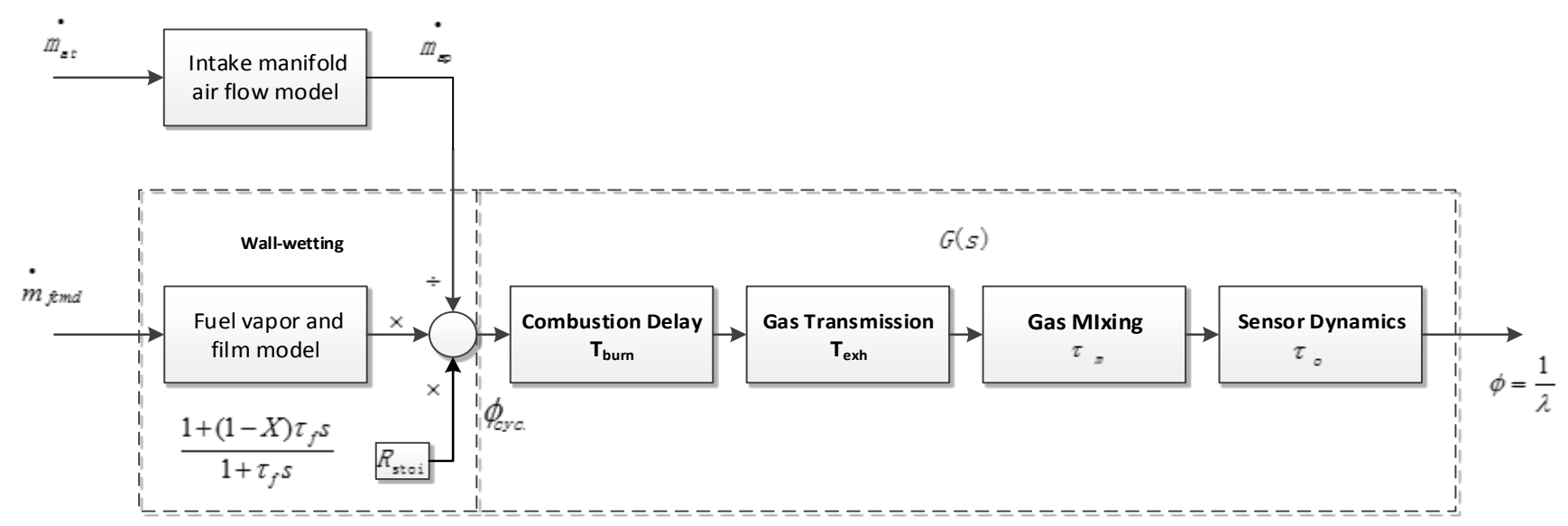

Fig. (1). AFR path dynamic model structure.

denotes the pressure just in front of the throttle plate after the air filter. It can also be shown that the total air mass flow past the throttle plate can be expressed as the product of a function of the throttle angle only and a function of the pressure ratio only. Although the model is nonlinear and complicate, it is convenient to fitting as the expression above.

As the pressure and temperature in the engine cylinder are not measured, it's difficult to use ideal gas law to calculate the real air mass in the cylinder. So volumetric efficiency $\left(e_{v}\right)$ is introduced to observe the amount of air in the cylinder by the pressure and temperature measured in the manifold. Using the speed density formula:

$\dot{\mathrm{m}}_{\mathrm{ap}}(t)=\frac{\mathrm{V}_{\mathrm{d}}}{120 R T_{m}}\left(e_{v} \bullet p_{m}\right) n$

Where $V_{d}$ is the engine displacement $(L), \mathrm{n}$ is the engine velocity (RPM), $p_{m}$ and $T_{m}$ are as above. Hendicks has simplified the equation as the linear in the manifold pressure:

$e_{v} \cdot p_{m}=s_{i} \cdot p_{m}-y_{i}$

Where $s_{i}, y_{i}$ are fitting parameters as constant and should not change much over the operating range of the engine [10].

So with the equations (3-7), the dynamic of air mass has been modelled and it's clearly that the air mass in the cylinder for fuel injection calculation can be observed by the throttle angel, manifold pressure and the engine velocity.

\subsection{Crank Shaft Dynamic Model}

Based on the energy conservation law, the change of the rotational kinetic energy is equal to the available acceleration power on the crank shaft. The physically expression is:

$\dot{n}=\frac{-\left(P_{f}+P_{p}+P_{b}\right)+H_{u} \dot{m}_{f y l l}\left(t-\tau_{d}\right) \eta_{i}}{I \cdot n}$
Where $I$ is the associate moment of inertia both of the engine and $\operatorname{load}\left(\mathrm{kg} \cdot \mathrm{m}^{2}\right), P_{f}, P_{p}, P_{b}$ are the friction, pumping losses and load power, $\tau_{d}$ is the delay between the change of speed and fuel flow step, $H_{u}$ is the fuel heating value( $J / \mathrm{kg}), \eta_{i}$.is the indicated efficiency which affected by the spark advance angle, lambda, crank shaft speed and manifold air pressure, the detail can be found in the paper [6].

\subsection{AFR Path Dynamic Model}

The close loop AFR control including the measurement data provided by the EGO(exhaust gas oxygen) sensor, the intake air mass estimate, and the fuel injection control. The dynamic of the AFR path comprise several delays, the wallwetting phenomenon, gas mixing dynamics and the sensor dynamics as showed in Fig. (1). The fuel and air model has been described in section 2.1 and 2.2.

AFR means the ratio of the air mass and fuel in the cylinder, it is commonly given by the variable of $\lambda$ which is the ratio of AFR and the stoichiometric ratio ( $R_{\text {stoi }}$, approximately 14.7 for gasoline). As the air flow is uncontrolled input, the equivalence ratio, $\varphi=\frac{1}{\lambda}$, is defined to make sure that the measurement is linear with the controlled input, fuel ,proportionally. So, the AFR path can be defined as:

$\phi_{c y l}=\frac{\dot{m}_{f c y l}(t) \bullet R_{\text {stoi }}}{\dot{m_{a p}}(t)}, \phi=G(s) \bullet \phi_{c y l}$

Where $G(s)$ includes all the overall dynamics of the AFR path from the gas and fuel mixed in the cylinder to the equivalence ratio measured by the sensor. There is a pure delay in the process $G(s)$ which includes the combustion time $\left(T_{\text {burn }}\right)$ and exhaust gas transportation $\left(T_{e x t}\right) . T_{\text {burn }}$ 


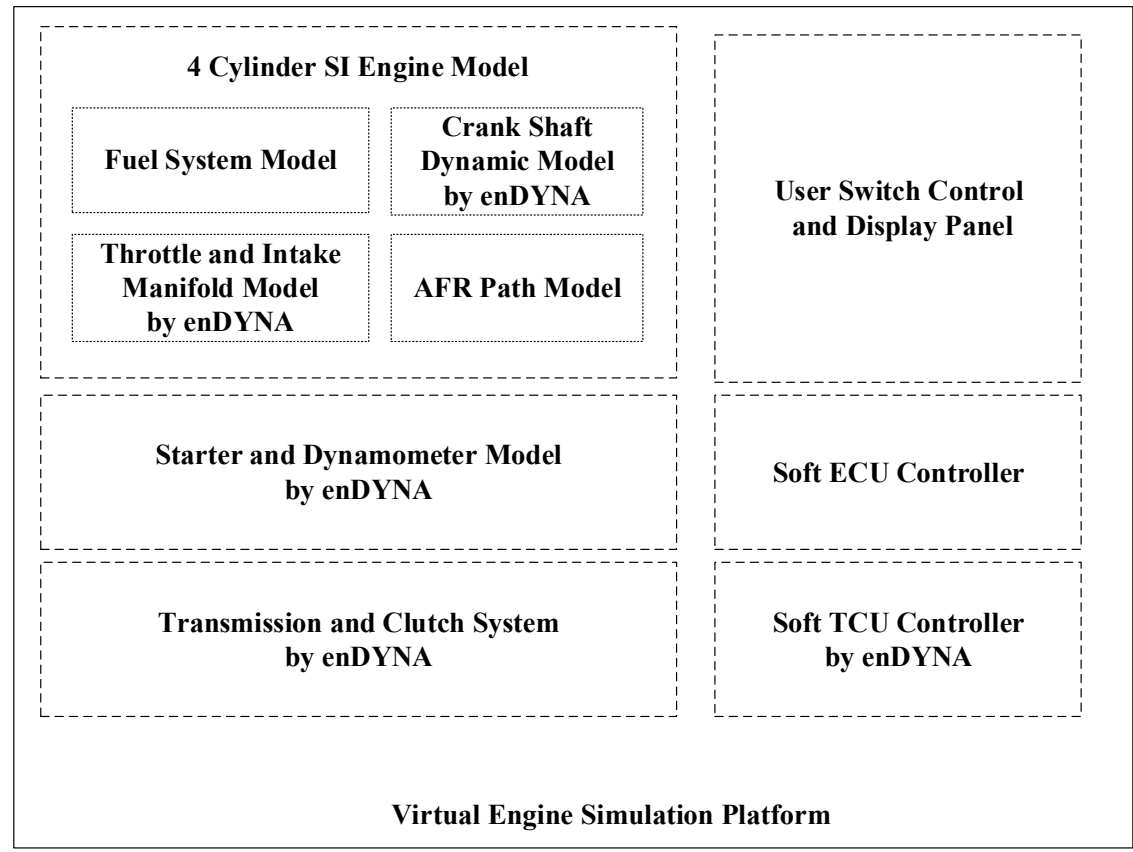

Fig. (2). Schematic of the virtual engine simulation platform.

indicates the time delay between the opening of inlet and exhaust valves, $T_{\text {ext }}$ indicates the time delay between the exhaust valve to the EGO sensor. The exhaust gas from each cylinder is mixed in the tailpipe and can be modelled as a first-order plant, the time constant is $\tau_{m}$. Meanwhile, EGO sensor can be modelled as a first-order plant, and the time constant $\tau_{o}$. is always $20 \mathrm{~ms}$ for the conventional sensor. Finally, $G(s)$ can be approximately expressed as:

$$
\left\{\begin{array}{c}
G(s)=e^{-\left(T_{\text {burn }}+T_{\text {ext }}\right) s} \frac{1}{\left(\tau_{m} s+1\right)\left(\tau_{o} s+1\right)} \\
T_{\text {burn }}=\frac{120(C Y L-1)}{n \bullet C Y L}, T_{e x t}=\frac{a}{\dot{m}_{a}}
\end{array}\right.
$$

Where $\mathrm{n}$ is the engine speed (RPM), CYL is the number of the cylinders, $\dot{m}_{a}$ is the air flow rate and $a$ is a fitting parameter based on the experimental data. More detail of the model can be find in the paper [11].

\section{VIRTUAL ENGINE SIMULATION PLATFORM}

Currently, HIL testing is used to shorten development and testing time for the engine control system, and running the deterministic engine model on the real-time simulator is an essential process in the application. In this section, an engine simulation platform is developed by MVEM theories for the real-time or off-line simulation and it is beneficial to the control strategy design according to the model-based method. And the process has actually become a standard procedure in the control system design of the automotive industry.
MVEM has been widely employed as a conventional model for the engine control practice. Both the identification and the parameter fitting methods have been extensively utilized in the engine modelling. In this work, the software package enDYNA which is based on the MVEM algorithms is employed for the real-time simulation of SI engines. As the consideration of more complicated factors and various possibilities, the commercial engine model is precise and reliable for the engine behavior representation. The enDYNA engine model is developed by TESIS and implemented in the Matlab/Simulink environment which supports the user defined model modification and allows the straightforward incorporation with the designed control algorithms.

In this work, enDYNA engine model is improved by adding the AFR dynamics described in Section 2. The schematic of the virtual engine simulation platform is shown in Fig. (2).

\subsection{Experiment Data Acquisition on Engine Test Bench}

In order to model a SGMW B15 engine, experiments for the operating data acquisition have been implemented on the engine test bench at Wuhan University of Technology. The geometry dimensions are listed in Table $\mathbf{1}$.

Table 1. SGMW B15 engine specifications.

\begin{tabular}{|c|c|}
\hline Engine Type & SI,4 cylinders, In-line \\
\hline \hline Displacement (liters) & $1.485 \mathrm{~L}$ \\
\hline Compression Ratio & $10.2: 1$ \\
\hline Bore(mm) & 74.7 \\
\hline Stroke(mm) & 84.7 \\
\hline Speed for maximum torque(rpm) & $3600-4000$ \\
\hline
\end{tabular}




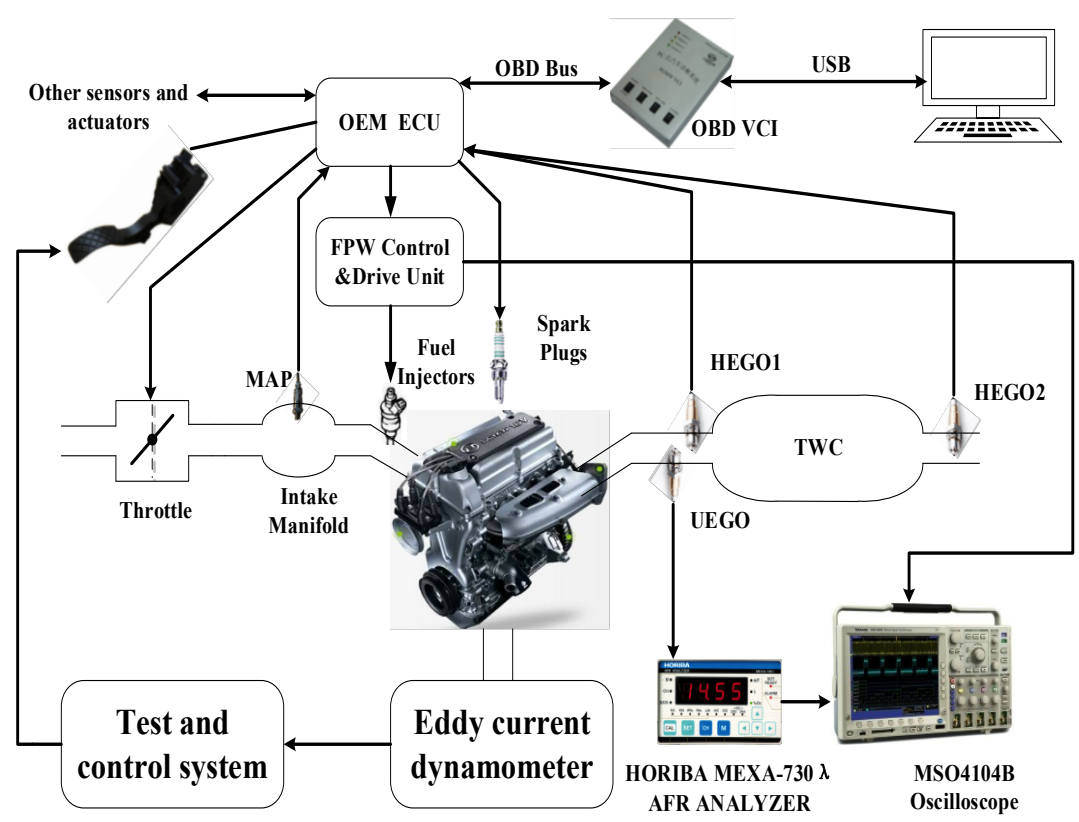

Fig. (3). Schematic of the engine test bench for data acquisition.

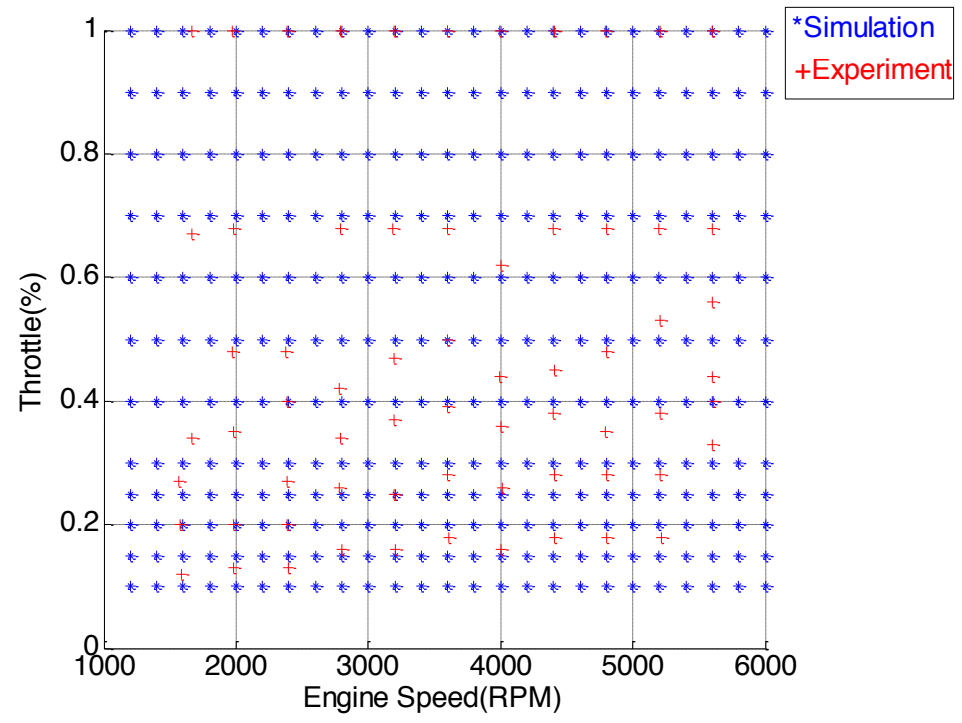

Fig. (4). The engine operation points for model validation.

As shown in Fig. (3), the SGMW B15 gasoline engine is connected with an eddy current dynamometer on the test bench. Engine velocity, torque, water temperature and other parameters of the test bench can be captured and displayed by the test and control system, and also the gas pedal is driven by it automatically. The engine is controlled by the corresponding OEM ECU which has been calibrated based on abundant experiment, and consequently, the measured data by the ECU is considered accurate enough in this work. The transient data of the sensors during the engine operation is acquired by the OBD VCI (Vehicle Communication Interface) developed at WHUT according to the diagnostics communication protocols in [12]. Furthermore, in order to identify the AFR path model, FPW Control \& Drive unit is developed for the fuel injection disturbance, and a UEGO is installed as near as possible to the HEGO1 as shown in Fig. (2). HORIBA MEXA-730 $\lambda$ AFR analyzer and MSO4104B oscilloscope are used to record the step response data after the FPW changed suddenly which would cover all the dynamic characteristics of the AFR path.

\subsection{Engine Model Validation}

The engine model matching approaches in this work is following the instruction of the guidance by TESIS [13], the data preprocessing tool is used for the parameter matching based on the measured data. For validating the precision of the simulation, the engine performance is evaluated by the comparison of the experiment and simulation results. In this work, the engine operation point is defined by the engine velocity and throttle angle as shown in Fig. (4). 66 sets of 


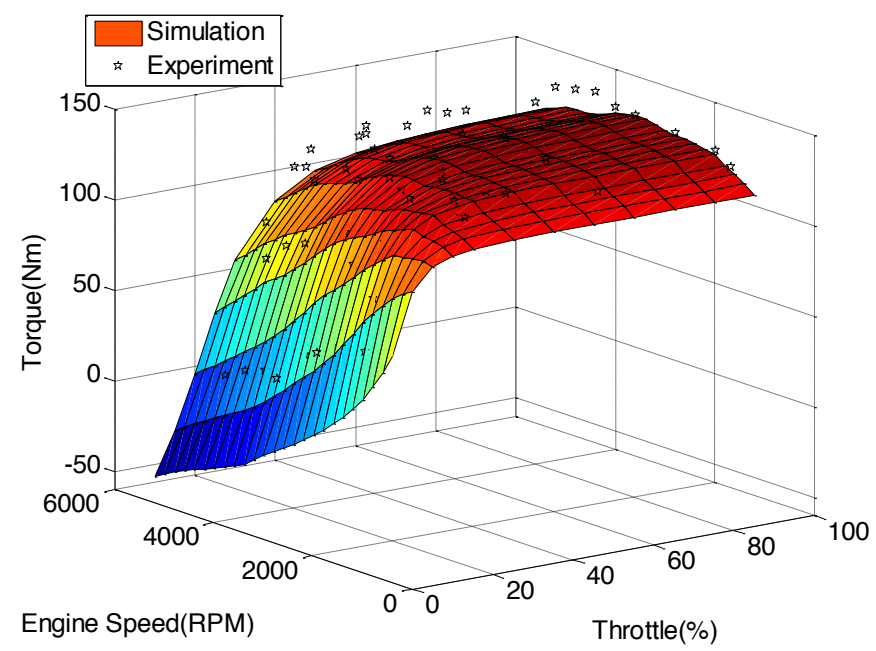

Fig. (5). The engine output torque validation by the simulation and experiment data.

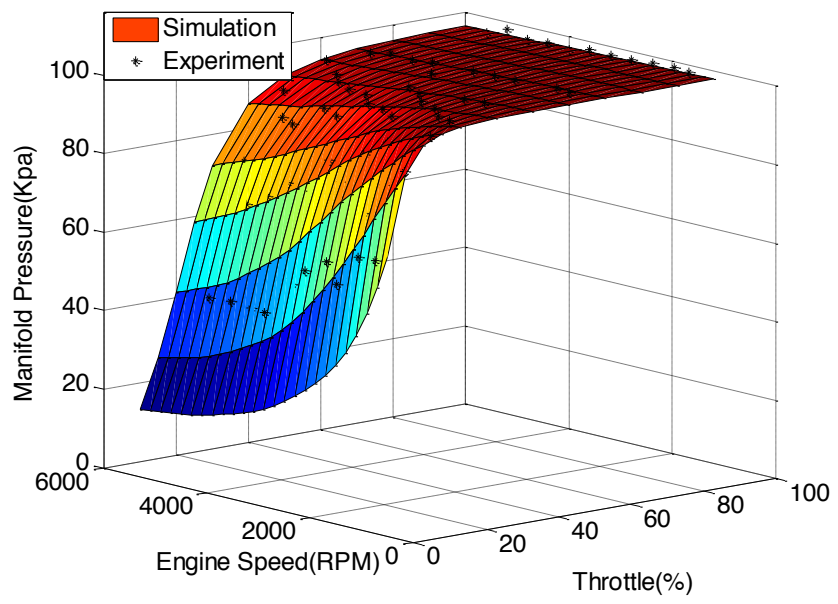

Fig. (6). The engine manifold pressure validation by the simulation and experiment data.

measured data have been acquired on the engine test bench and 300 sets of simulation data have been obtained by the simulation model. At each different operation point, the data is recorded at the steady state. However, as the physical phenomenon and the limits of the test and control system, the test points of the experiment distribute asymmetrically.

First, the engine output torque is validated as shown in Fig. (5) and some of experiment data hides under the surface of the simulation result. It has been shown that the maximum torque is generated around 4000 RPM as the same as the engine specification and the test shows a satisfactory performance of the engine at steady state mode. The output torque is negative at high speed because of the reversed towing by the dynamometer in the simulation and this situation is not covered in the experiments.

On the B15 engine, MAP sensor is employed for the estimation of the intake air mass which significantly impact on the AFR control. Also, the manifold pressure is displayed in Fig. (6) for comparison purpose. In the situation of high engine velocity and light load, the air pressure in the intake manifold is low. Also, it changes little and is almost close to the atmospheric pressure when the throttle angle is large. It's obviously shown that simulation result is consistent with the experiment.

For the AFR path model verification, small step wave fuel flow perturbations is used for the transient response analysis. The small step wave is superimposed on the constant fuel flow command at steady state and the modulation depth is about $10 \%$ of the constant. As described in Fig. (1), the engine velocity and the air mass remain steady, the effect of the fuel flow command perturbation should impact the lambda signal measured by the AFR analyzer and it would cover the whole dynamics of AFR path. On the engine test bench, the HEGO is removed to guarantee the OEM ECU runs in the open loop.

The injection pulse width signal is recorded by the oscilloscope and then be calculated to the fuel command step. To eliminate the effect of index dimension and quantity of data, the calculated fuel command, $\mathrm{X}$, is normalized in the following way: 


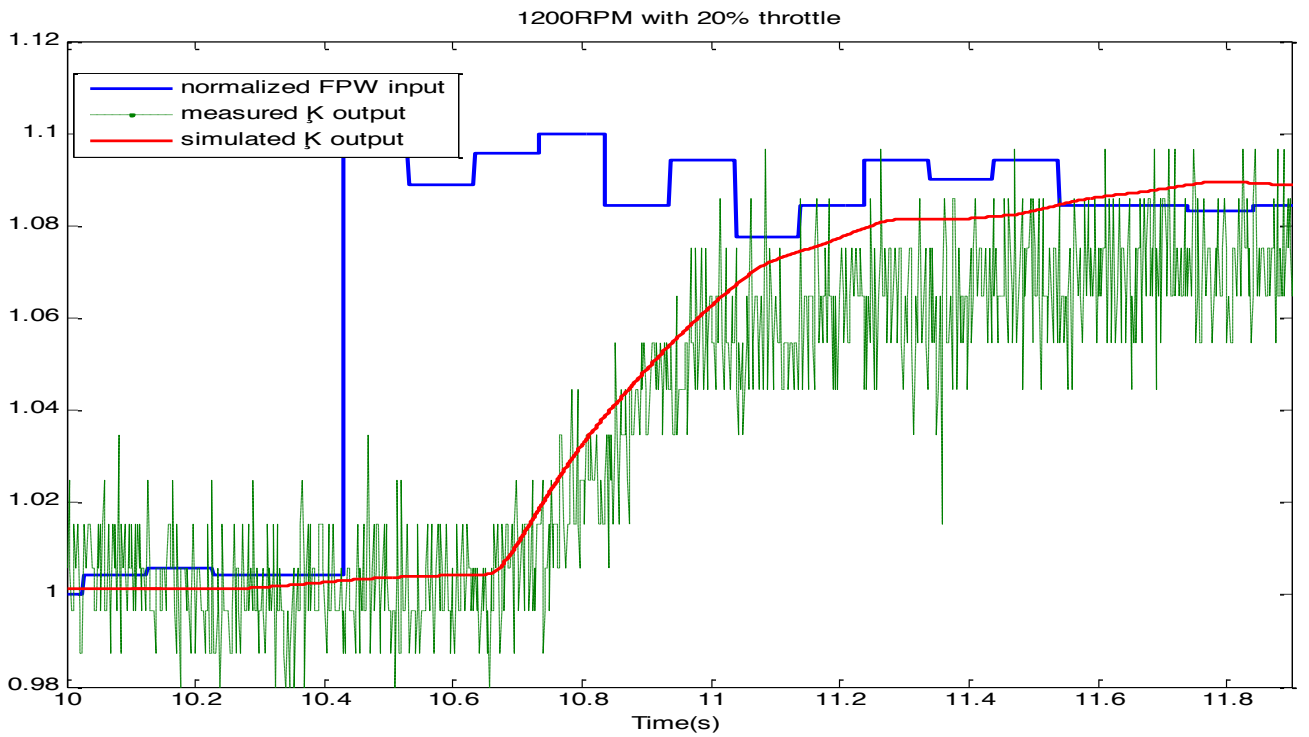

Fig. (7). Comparison of measured and simulated $\varphi$ signal with small step wave fuel flow perturbations at $1200 \mathrm{RPM}$ with $20 \%$ throttle.

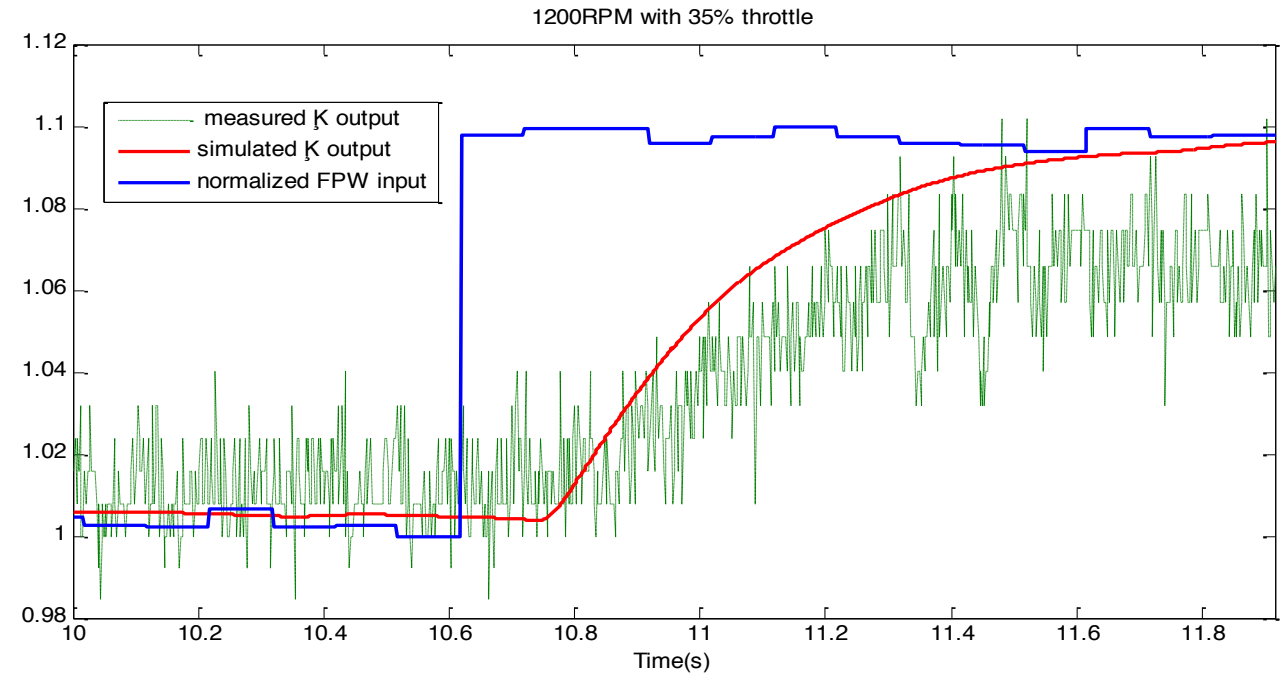

Fig. (8). Comparison of measured and simulated $\varphi$ signal with small step wave fuel flow perturbations at $1200 \mathrm{RPM}$ with $35 \%$ throttle.

$\bar{X}=\frac{X-X_{\text {min }}}{X_{\text {max }}-X_{\text {min }}} \times 0.1+1$, so: $\bar{X} \subset[1,1.1]$

The fuel command in the simulation multiplies by the normalized FPW input, $\bar{X}$, to establish the stimulus signal in the simulation model to result in the same characteristic of the experiment perturbations. The measured $\varphi$ signal and the simulation response at different operating points are shown in Fig. (7), Fig. (8) and Fig. (9). The step perturbation is implement when the engine is at steady state and all of the data is synchronized by the time.

With the comparison of the results, the time delay and system dynamics is obviously presented. The fluctuation of the normalized FPW input is due to the open loop control of OEM ECU as the operating points is unable to keep at abso- lute steady state by the eddy current dynamometer. The measured $\varphi$ signal by the AFR analyzer is also affected by the noise and systemic error which is neglected in this paper. The experimental and simulation results are demonstrate that the model described in Section 2.4 is appropriate for the AFR path dynamic modelling.

\section{DESIGN OF SOFT ECU FOR ENGINE SIMULA- TION MODEL}

Although the ECU emulator block is provided in enDYNA for the off-board simulation, it's hard to modify the control method and parameter because of the packaged block. A soft ECU controller model is designed for the control of the engine simulation model as shown in Fig. (10). The input and output signals are connected with the corre- 


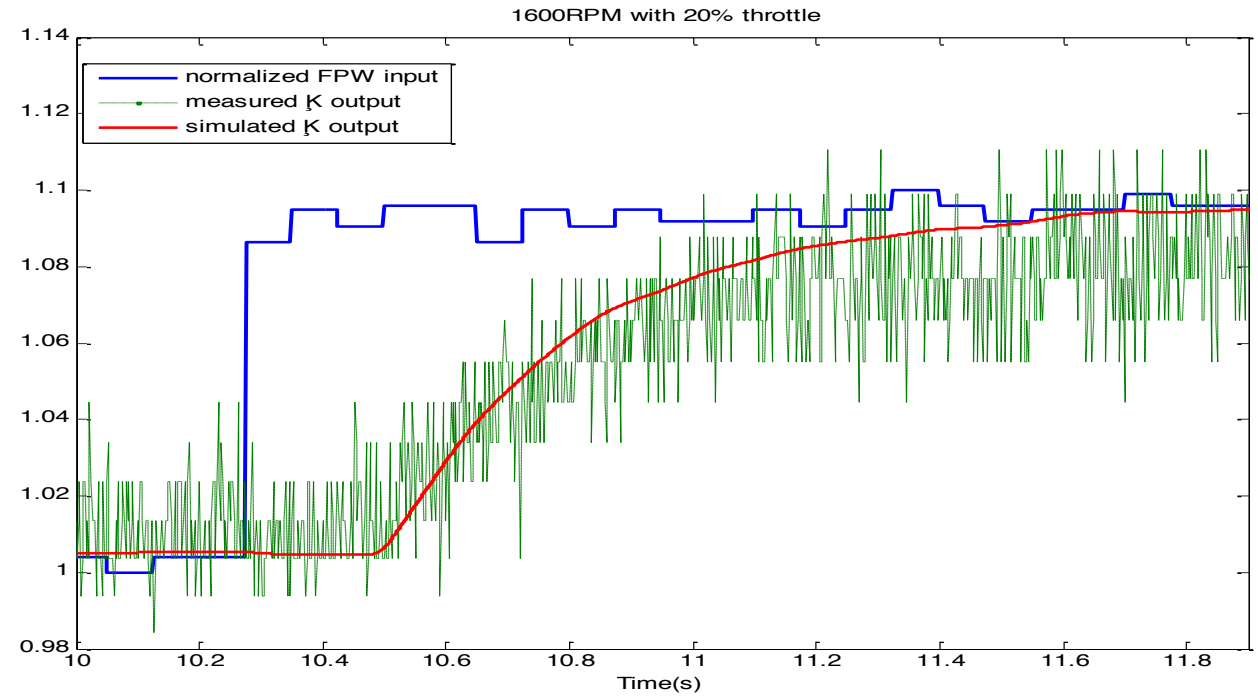

Fig. (9). Comparison of measured and simulated $\varphi$ signal with small step wave fuel flow perturbations at 1600 RPM with $20 \%$ throttle.

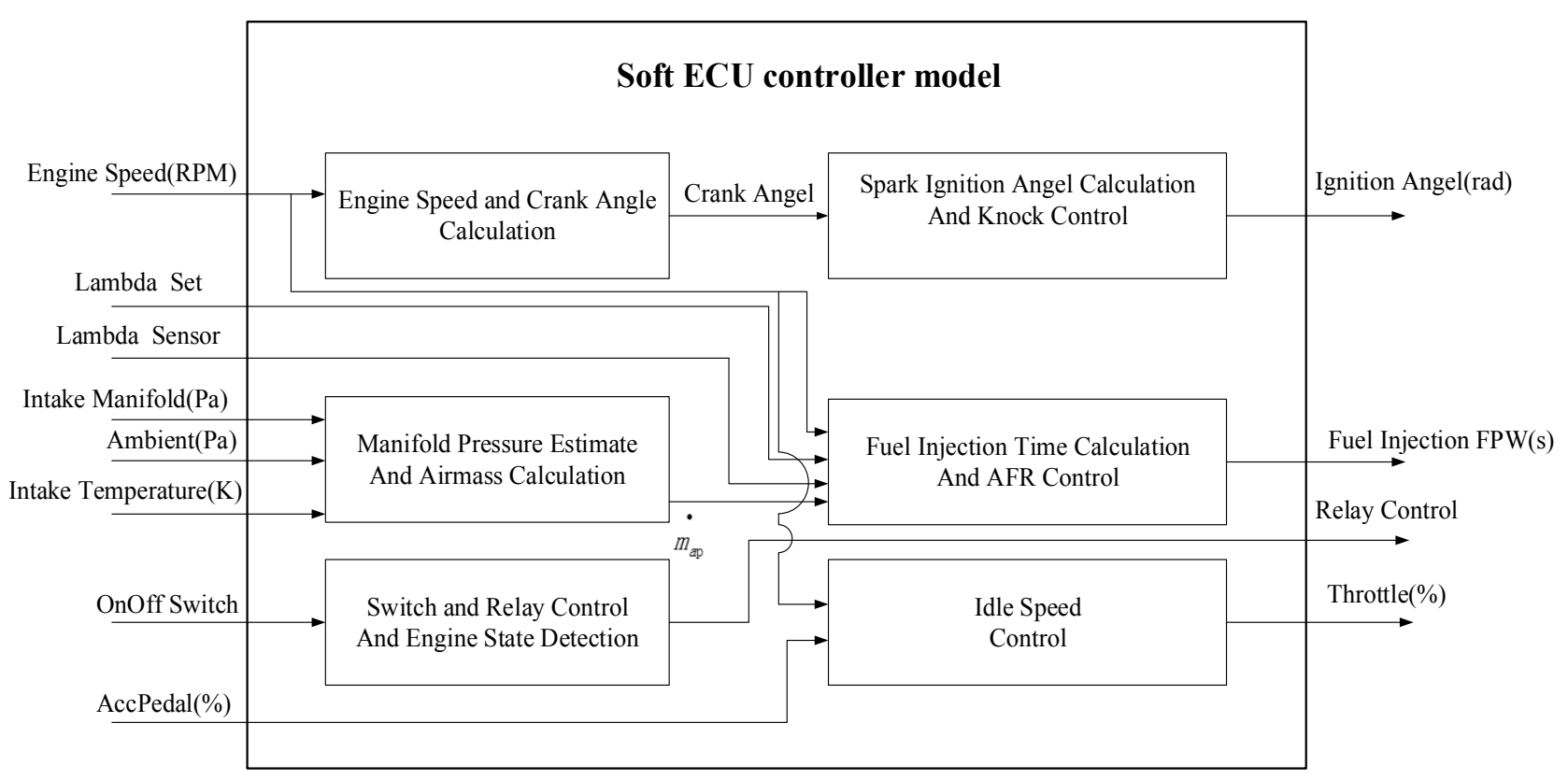

Fig. (10). The architecture of the soft ECU controller model.

sponding SGMW B15 engine model described above. The equations in Section 2.2 is used for the intake manifold air mass estimation and calculation. Optimized spark advance angle data is initialized based on the experiment and saved in the lookup table.

When the engine speed is below the intended speed and the gas-pedal angle is smaller than the limit, idle speed control block is activated for the automatic throttle control.

\subsection{Simulation Results}

As the enDYNA model only emphasize on the basic engine function realization, AFR dynamic is added as described in Section 2 to improve the engine model for AFR control application. To demonstrate the simulation results of the B15 engine, two simulation test were carried out as shown in Fig. $(\mathbf{1 1}, \mathbf{1 2})$.

In Fig. (11), the simulation was set at driving mode means the engine performed under the control of the gas pedal without the load torque from dynamometer. Idle speed state is not included in this case so that the throttle position followed the movement of the gas pedal. It was obviously to see that the AFR signal fluctuated with the throttle angle which would greatly influence the intake air mass and the suddenly adjusted fuel injection would not be drawn into the cylinder due to the wall-wetting phenomenon as seen in section 2.1. At the steady state, the throttle angle changed slowly so that the AFR could be kept around 1 although the en- 


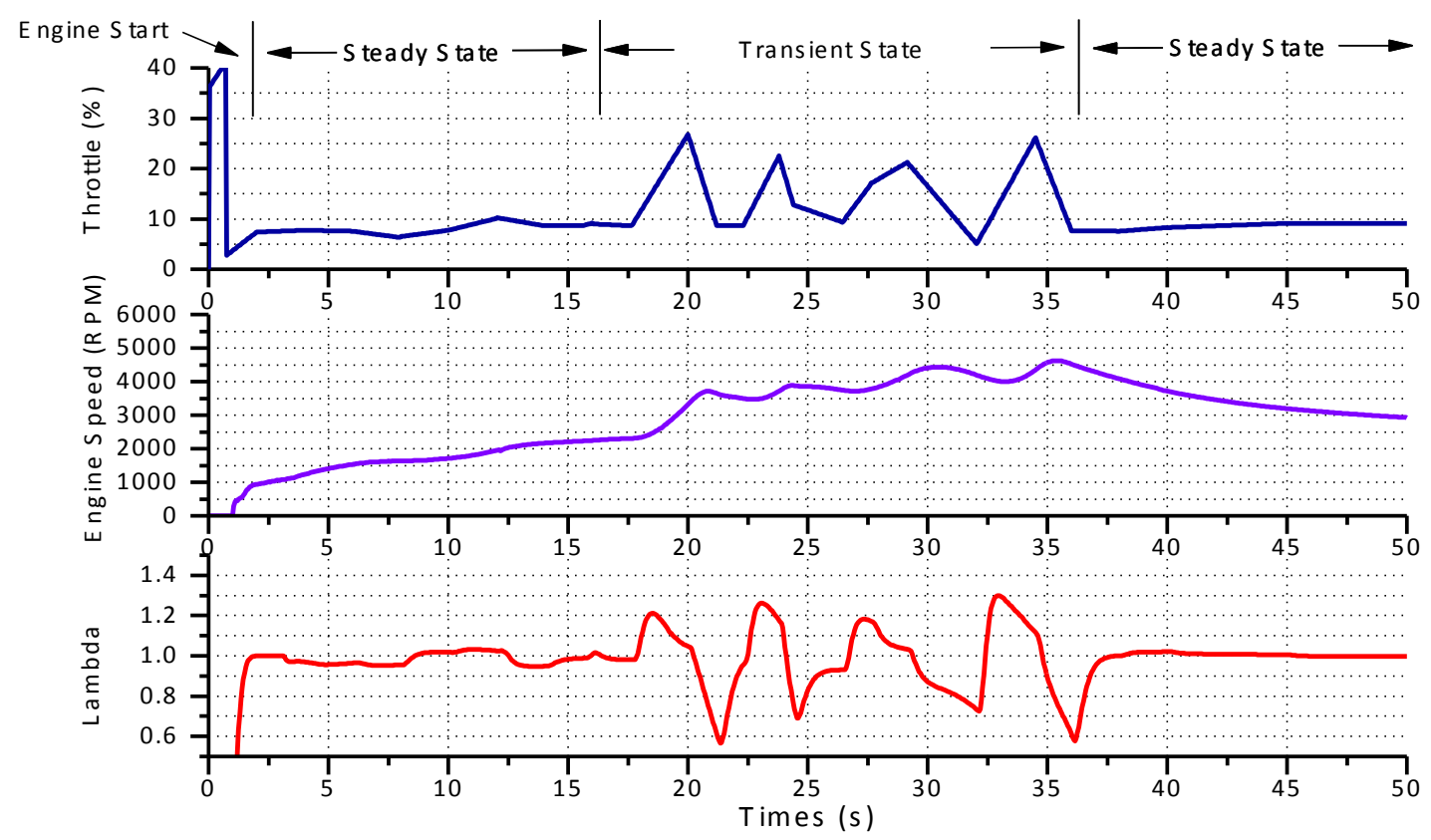

Fig. (11). Engine model performance at transient and steady state with open loop AFR control.

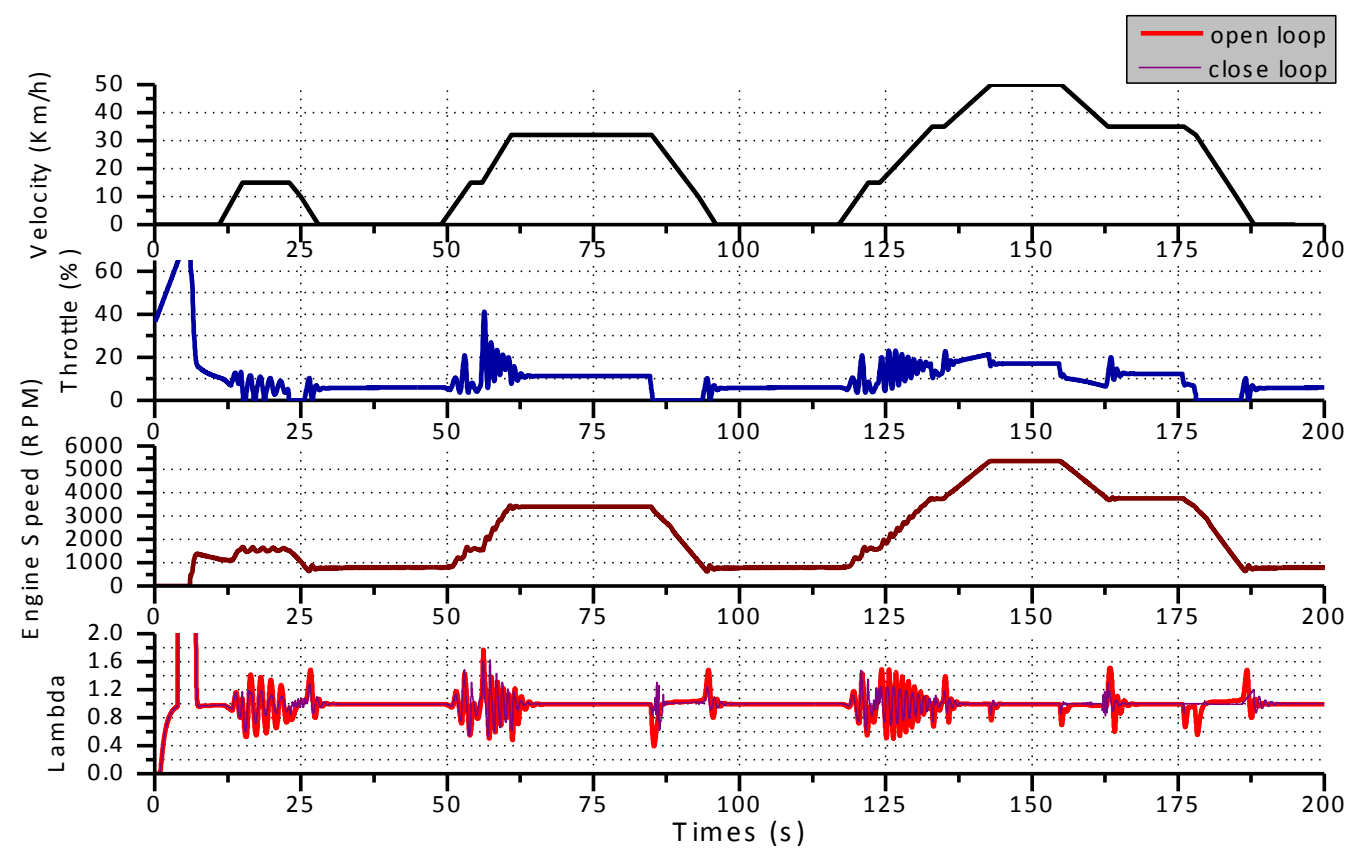

Fig. (12). The comparison of AFR control with open loop AFR and close loop under the ECE driving cycle.

gine speed changed; but, the deviation was large at the transient state because the error caused by delay and system dynamics was not able to compensate with the open loop control.

For comparison purposes, a simulation was carried out using open loop and close loop controller at the same driving states as shown in Fig. (12). ECE-15 driving cycle was employed for the model test because it contained 15 kinds of driving mode such as idle speed, acceleration, constant speed and decelerate state of the engine. The gas pedal was controlled by the enDYNA soft tool automatically in the simulation to achieve the expected velocity during the driving cycle and the transmission was set at the first gear to cover the whole operating points of the engine in the test. Idle speed control was active when the engine speed below 800RPM. As there is no load torque from the dynamometer to manipulate the engine speed in such kind of test, the throttle was adjusted quickly to maintain the engine speed at the fixed acceleration according to the driving cycle, so the AFR signal also has the large deviation as seen in Fig. (12). A PID 
controller was designed for the AFR close loop control which compensated fuel injection according to the equivalence ratio error with the measured from the UEGO sensor model. It was obviously to see that the overshoot of the AFR signal could be suppressed. But, the gains of the PID feedback controller cannot be made aggressive to maintain stability. Moreover, since the fuel injection reacted compensation from the feedback path only after the delay, the overshoot in the response was difficult to avoid by any classic controller.

\section{CONCLUSION}

In this paper, the analytical MVEM with AFR path dynamics has been discussed in order to model the SI engine for AFR control application. The enDYNA software tool is used for the SI engine modelling and be improved by adding the AFR dynamics. Experiments on the engine test bench have been done for the data acquisition which is used for the engine model matching. In comparison of the experiment results, the simulation model is validated and the results demonstrate that the model is appropriate for the $\mathrm{B} 15$ engine. A soft ECU controller model is designed to replace the ECU emulator in the simulation as the other control algorithm can be implemented is the simulation. Two simulations results has been presented to show the engine performance at different operating points. It has also been observed that the PID controller can suppress the overshoot during the throttle position varying.

As the wall-wetting phenomenon and AFR dynamics exist, avoiding the AFR signal overshoot in the response is still challenging due to the delay and parameter varying. The simulation model reported here can be used as the virtual engine control object in the AFR control application. Further control algorithm to deal with the delay and parameter varying problems for AFR regulation are currently under investigation.

\section{CONFLICT OF INTEREST}

The authors confirm that this article content has no conflicts of interest.

\section{ACKNOWLEDGEMENTS}

The authors acknowledge the support of International S\&T Cooperation Program of China (NO.2012DFA11180) and the Fundamental Research Funds for the Central Universities (WUT:2014-IV-076).

\section{REFERENCES}

[1] N. E. Kahveci and M. J. Jankovic, "Adaptive controller with delay compensation for Air-Fuel Ratio regulation in SI engines," In American Control Conference (ACC), 2010, 2010, pp. 2236-2241.

[2] E. Hendricks, T. Vesterholm, P. Kaidantzis, P. Rasmussen and M. Jensen, "Nonlinear Transient Fuel Film Compensation (NTFC)," SAE Technical Paper (930767), 1993, doi: 10.4271/930767.

[3] J. D. Powell, N. P. Fekete and C. Chen-Fang, "Observer-based air fuel ratio control," IEEE Control Systems, vol. 18, pp. 72-83, 1998.

[4] L. Guzzella and C. H. Onder, Introduction to Modeling and Control of Internal Combustion Engine Systems Springer: Berlin Heidelberg, vol. 25, 2010.

[5] E. Hendricks, "Engine modelling for control applications: a critical survey - springer," Meccanica, vol. 32, pp. 387-396, 1997.

[6] E. Hendricks and S. Spencer, "Mean Value Modelling of Spark Ignition Engines". SAE Technical Paper (900616), 1990.

[7] J. D. Powell, N. P. Fekete and C. Chen-Fang, "Observer-based air fuel ratio control," IEEE Control Systems, vol. 18, pp. 72-83, 1998.

[8] A. White, Z. Guoming and C. Jongeun, "Hardware-in-the-loop simulation of robust gain-scheduling control of port-fuel-injection processes," IEEE Transactions on Control Systems Technology, vol. 19, pp. 1433-1443, 2011.

[9] R. Isermann, J. Schaffnit and S. Sinsel, "Hardware-in-the-loop simulation for the design and testing of engine-control systems," Control Engineering Practice, vol. 7, pp. 643-653, 1999.

[10] E. Hendricks, A. Chevalier, M. Jensen, S.C. Sorenson, D. Trumpy and J. Asik, "Modelling of the Intake Manifold Filling Dynamics". SAE Technical Papers (960037).1996.

[11] U. Kiencke and L. Nielsen, Automotive Control Systems: For Engine, Driveline, and Vehicle: Springer: Berlin Heidelberg, 2005.

[12] J. Hu, F. Yan, J. Tian, P. Wang, and K. Cao, "Developing PCBased Automobile Diagnostic System Based on OBD System,", Chengdu, China, IEEE Power and Energy Society (PES); State Grid of China; Siemens Ltd.; Sichuan University; Chongqing University, 2010.

[13] TD_endyna_preprocessing_tutorial: Available from: http://www.tesis-dynaware.com/en.html

\footnotetext{
Received: November 28, 2014

Revised: January 09, 2015

Accepted: January 20, 2015

(C) Lei et al.; Licensee Bentham Open.

This is an open access article licensed under the terms of the Creative Commons Attribution Non-Commercial License (http://creativecommons.org/licenses/by-nc/3.0/) which permits unrestricted, non-commercial use, distribution and reproduction in any medium, provided the work is properly cited.
} 